\title{
REAÇÕES DE 1,2-DICLORO-4,5-DINITROBENZENO COM AMINAS. MONOSSUBSTITUIÇÃO DE NITRO E DISSUBSTITUIÇÃO DE CLORO E NITRO
}

\author{
Fabrício Gava Menezes, Juan Ricardo, Rafael Dias, Adailton J. Bortoluzzi e César Zucco* \\ Departamento de Química, Universidade Federal de Santa Catarina, CP 476, 88040-900 Florianópolis - SC, Brasil
}

Recebido em 1/2/06; aceito em 16/5/06; publicado na web em 26/9/06

\begin{abstract}
REACTIONS OF 1,2-DICHLORO-4,5-DINITROBENZENE WITH AMINES: MONOSUBSTITUTION OF CHLORINE AND DISUBSTITUTION OF CHLORINE AND NITRO. 1,2-dichloro-4,5-dinitrobenzene (DCDNB) reacts with primary and secondary amines, in acetonitrile, at room temperature, to give a monosubstituted nitro product with a yield of 85 to $95 \%$. The chloro-nitrodisubstituted product is formed with excess amine under reflux. Piperidine, pyrroline, dimethylamine and methylamine were the most reactive reagents in both mono- and disubstitution.
\end{abstract}

Keywords: 1.2-dichloro-4,5-dinitrobenzene; amines; nucleophilic aromatic substitution.

\section{INTRODUÇÃO}

É comum encontrar-se nos livros textos de Química Orgânica que a substituição nucleofílica em anéis aromáticos é muito lenta e, por isso, não só impraticável, mas sem interesse sintético. No entanto, entende-se que devem ser excluídas dessa assertiva reações envolvendo substratos altamente ativados por grupos elétronatraentes (mecanismo $\mathrm{S}_{\mathrm{N}} \mathrm{Ar}$ ); catalisadas por bases fortes (mecanismo via intermediário benzino); nas quais o nitrogênio de um sal de diazônio é substituído por um nucleófilo (mecanismo $\mathrm{S}_{\mathrm{N}} 1$ ) e iniciadas por doadores de elétrons (mecanismo via radical livre). Essas reações podem ocorrer em condições suaves e, em cada reação, opera um mecanismo diferente, conforme explicitado ${ }^{1,2}$.

O 1,2-dicloro-4,5-dinitrobenzeno, DCDNB, com quatro grupos elétron-atraentes, reage com o íon hidroxila, em água ou em misturas de DMSO:água, para formar 4,5-dicloro-2-nitrofenol, por meio da substituição de um grupo nitro. Foi postulado para essa reação o mecanismo SET ("single electron transfer"), cujas evidências maiores foram obtidas por RMN de hidrogênio ${ }^{3,4}$. O produto da reação entre DCDNB e $n$-butilamina, em éter etílico, 1,2-dicloro-4(n-butilamino)-5nitrobenzeno, também é resultado da substituição de um grupo nitro ${ }^{5}$. Todavia, a reação de DCDNB com morfolina, em DMSO, formou, com $68 \%$ de rendimento, 1-cloro-2,4-dimorfolino-5-nitrobenzeno, resultado da dissubstituição de um cloro e de um nitro ${ }^{6}$.

Essas reações do $\mathrm{DCDNB}^{3,5,6}$ apresentam rendimento variando entre 68 a $90 \%$ e são facilmente realizadas, porém, os resultados não foram previsíveis em relação ao tipo de substituição. A formação de produtos mono e dissubstituídos exige que novas investigações sejam realizadas para se determinar as condições em que um nitro ou um nitro e um cloro são substituídos. Além disso, é necessário que seja esclarecido se os compostos mono e dissubstituídos são formados simultaneamente ou se o dissubstituído é formado a partir do mono. Sabe-se que o grupo nitro ativa anéis aromáticos para as reações do tipo $\mathrm{S}_{\mathrm{N}} \mathrm{Ar}^{1,2}$, sendo um grupo de saída bastante comum sob diversas condições ${ }^{1,7,8}$. O cloro, por sua vez, é um ativador mais fraco e comumente conhecido como bom grupo de saída, porém, menos lábil que o nitro ou o flúor, em várias reações de substituição nucleofílica aromática ${ }^{1,7}$.
O presente trabalho diz respeito às reações de preparação de derivados do DCDNB com aminas primárias e secundárias. Essas duas séries de aminas mostram como varia o comportamento da reação em função da nucleofilicidade do reagente, dos efeitos estéricos, da temperatura e, inclusive, do solvente, possibilitando o entendimento das condições em que o nitro ou o cloro e o nitro atuam simultaneamente como grupo de saída.

\section{PARTE EXPERIMENTAL}

Os pontos de fusão, medidos em um aparelho MQAPF - 301, Microquímica, não foram corrigidos. Os espectros de RMN de ${ }^{1} \mathrm{H}$ foram obtidos em um aparelho Variam, $400 \mathrm{MHz}$, e em um aparelho Brucker, FT $200 \mathrm{MHz}$, utilizando $\mathrm{CDCl}_{3}$ como solvente. Os valores de deslocamento químico $(\delta)$ são dados em ppm e as constantes de acoplamento $(J)$ em Hz. As análises de CHN foram realizadas em um analisador elementar CHNS-O, Carlo Erba, modelo E-1110. As estruturas cristalográficas foram determinadas em difratômetro Enraf-Nonius CAD.

O DCDNB, Aldrich, foi utilizado sem qualquer purificação prévia. As aminas foram adquiridas comercialmente e destiladas antes das reações, exceto metilamina e dimetilamina, que foram utilizadas na forma de solução aquosa. Os demais reagentes foram obtidos comercialmente e utilizados sem purificação.

\section{Procedimento 1: obtenção de produtos mono-nitro-substituídos}

Em um frasco de vidro de $10 \mathrm{~mL}$ foi adicionado $0,10 \mathrm{~g}(0,42$ mmol) de DCDNB a uma solução de 1,26 mmol da amina (metilamina, $n$-propilamina, $i$-propilamina, $n$-butilamina, $t$ butilamina, cicloexilamina, benzilamina, anilina, dimetilamina, dietilamina, di-n-propilamina, $n$-butilmetilamina, piperidina e pirrolidina) em $3 \mathrm{~mL}$ de acetonitrila, em temperatura ambiente. As reações foram acompanhadas por cromatografia de camada delgada com diclorometano-hexano 1:1,2 (v/v). Após o consumo do DCDNB, o solvente foi evaporado em rotavapor. O produto, quando sólido, foi recristalizado em etanol e lavado com etanol frio e água. Quando o produto era um óleo, foi purificado por coluna flash, utilizando-se sílica-gel 60 como fase estacionária e uma mistura de diclorometano-hexano 1:3 (v/v), como eluente. 
1,2-dicloro-4-metilamino-5-nitrobenzeno, 1: p.f. $146{ }^{\circ} \mathrm{C}\left(\mathrm{Lit}^{9}\right.$ $\left.148{ }^{\circ} \mathrm{C}\right)$; RMN de ${ }^{1} \mathrm{H}(400 \mathrm{MHz}) \delta: 8,29(\mathrm{~d}, 1 \mathrm{H}, \mathrm{ArH}, J=2), 7,91$ (sl, 1H, NH), 6,97 (d, 1H, ArH, $J=2), 3,02\left(\mathrm{dd}, 3 \mathrm{H}, \mathrm{CH}_{3}, J=2 \mathrm{e}\right.$ $5,2)$; rendimento $89 \%$.

1,2-dicloro-4(n-propilamino)-5-nitrobenzeno, 2: p.f. $83-84{ }^{\circ} \mathrm{C}$ (Lit. $\left.{ }^{9}: 84-85{ }^{\circ} \mathrm{C}\right)$; RMN de ${ }^{1} \mathrm{H}(200 \mathrm{MHz}) \delta$ : 8,28 (s, 1H, ArH), 7,97 (sl, 1H, NH), 6,97 (s, 1H, ArH), 3,24 (m, 2H, CH $), 1,72(\mathrm{~m}, 2 \mathrm{H}$, $\mathrm{CH}_{2}$ ), 1,06 (t, $3 \mathrm{H}, \mathrm{CH}_{3}, J=7,4$ ); análise elementar calculada para $\mathrm{C}_{9} \mathrm{H}_{10} \mathrm{Cl}_{2} \mathrm{~N}_{2} \mathrm{O}_{2}$ : C, 43,40; $\mathrm{H}, 4,05 ; \mathrm{N}, 11,25$; encontrada: $\mathrm{C}, 43,44$; $\mathrm{H}, 4,10$; $\mathrm{N}, 11,28$; rendimento: $92 \%$.

1,2-dicloro-4(i-propilamino)-5-nitrobenzeno, 3: p.f. $100-101{ }^{\circ} \mathrm{C}$ (lit. ${ }^{10} 100{ }^{\circ} \mathrm{C}$ ); RMN de ${ }^{1} \mathrm{H}(200 \mathrm{MHz}) \delta: 8,27$ (s, 1H, ArH), 7,94 (sl, 1H, NH), 6,97 (s, 1H, ArH), 3,77 (m, 1H, CH), 1,34 (d, 6H, $\left.\mathrm{CH}_{3}, J=6,4\right)$; análise elementar calculada para $\mathrm{C}_{9} \mathrm{H}_{10} \mathrm{Cl}_{2} \mathrm{~N}_{2} \mathrm{O}_{2}: \mathrm{C}$, 43,40; H, 4,05; N, 11,25; encontrada: C, 43,48; H, 4,09; N, 11,26; rendimento: $95 \%$.

1,2-dicloro-4(n-butilamino)-5-nitrobenzeno, 4: p.f. $63{ }^{\circ} \mathrm{C}$; $\mathrm{RMN}$ de ${ }^{1} \mathrm{H}(400 \mathrm{MHz}) \delta: 8,26(\mathrm{~d}, 1 \mathrm{H}, \mathrm{ArH}, J=2), 7,98(\mathrm{sl}, 1 \mathrm{H}, \mathrm{NH})$, 6,96 (s, 1H, ArH), 3,27 (m, 2H, $\left.\mathrm{CH}_{2}\right), 1,72\left(\mathrm{~m}, 2 \mathrm{H}, \mathrm{CH}_{2}\right), 1,49$ (m, $\left.2 \mathrm{H}, \mathrm{CH}_{2}\right), 0,99\left(\mathrm{t}, 3 \mathrm{H}, \mathrm{CH}_{3}, J=7,2\right)$; análise elementar calculada para $\mathrm{C}_{10} \mathrm{H}_{12} \mathrm{Cl}_{2} \mathrm{~N}_{2} \mathrm{O}_{2}: \mathrm{C}, 45,65 ; \mathrm{H}, 4,60 ; \mathrm{N}, 10,65$; encontrada: $\mathrm{C}$, 45,68; H, 4,66; N, 10,61; rendimento: $92 \%$.

1,2-dicloro-4(t-butilamino)-5-nitrobenzeno, 5: p.f. $110^{\circ} \mathrm{C}$; RMN de ${ }^{1} \mathrm{H}(200 \mathrm{MHz}) \delta: 8,29$ (sl, 2H, ArH e NH sobrepostos), 7,21 (s, $1 \mathrm{H}, \mathrm{ArH}), 1,50\left(\mathrm{~s}, 9 \mathrm{H}, \mathrm{CH}_{3}\right)$; análise elementar calculada para $\mathrm{C}_{10} \mathrm{H}_{12} \mathrm{Cl}_{2} \mathrm{~N}_{2} \mathrm{O}_{2}: \mathrm{C}, 45,65 ; \mathrm{H}, 4,60 ; \mathrm{N}, 10,65$; encontrada: $\mathrm{C}, 45,70$; $\mathrm{H}, 4,64 ; \mathrm{N}, 10,64$; rendimento: $94 \%$.

1,2-dicloro-4-cicloexilamino-5-nitrobenzeno, 6: p.f. 103-104 ${ }^{\circ} \mathrm{C}$; RMN de ${ }^{1} \mathrm{H}(200 \mathrm{MHz}) \delta: 8,28$ (s, $\left.1 \mathrm{H}, \mathrm{ArH}\right), 8,03$ (sl, 1H, NH), 6,97 (s, 1H, ArH), 3,45 (m, 1H, CH), 2,18-1,40 (m, 4H, $\left.\mathrm{CH}_{2}\right), 1,30-$ $1,50\left(\mathrm{~m}, 6 \mathrm{H}, \mathrm{CH}_{2}\right)$; análise elementar calculada para $\mathrm{C}_{12} \mathrm{H}_{14} \mathrm{~N}_{2}: \mathrm{C}$, 49,84; H, 4,88; N 9,69; encontrada: C, 49,93; H, 4,96; N, 9,67; rendimento: $92 \%$.

1,2-dicloro-4-benzilamino-5-nitrobenzeno, 7: p.f. $110-111{ }^{\circ} \mathrm{C}$ (Lit. ${ }^{9} 104{ }^{\circ} \mathrm{C}$ ); RMN de ${ }^{1} \mathrm{H}(200 \mathrm{MHz}) \delta: 8,32$ (sl, 1H, NH ), 8,28 (s, 1H, ArH), 7,43-7,28 (m, 5H, ArH), 6,96 (s, 1H, ArH), 4,49 (d, $\left.2 \mathrm{H}, \mathrm{CH}_{2}, J=12,7\right)$; análise elementar calculada para $\mathrm{C}_{13} \mathrm{H}_{10} \mathrm{Cl}_{2} \mathrm{~N}_{2} \mathrm{O}_{2}$ : C, 52,55; H, 3,39; N, 9,43; encontrada: C, 52,59; H, 3,44; N, 9,41; rendimento: $85 \%$.

1,2-dicloro-4-anilineo-5-nitrobenzeno, 8: p.f. $92-93{ }^{\circ} \mathrm{C}$ (Lit. ${ }^{9}$ $\left.96{ }^{\circ} \mathrm{C}\right) ; \mathrm{RMN}$ de ${ }^{1} \mathrm{H}(400 \mathrm{MHz}) \delta: 9,43(\mathrm{sl}, 1 \mathrm{H}, \mathrm{NH}), 8,32(\mathrm{~s}, 1 \mathrm{H}$, ArH), 7,45 (m, 2H, ArH), 7,27 (m, 4H, ArH); rendimento: $54 \%$.

1,2-dicloro-4-dimetilamino-5-nitrobenzeno, 9: p.f. 99-101 ${ }^{\circ} \mathrm{C}$ $\left(\mathrm{Lit}^{11} 103-104{ }^{\circ} \mathrm{C}\right)$; RMN de ${ }^{1} \mathrm{H}(400 \mathrm{MHz}) \delta$ : 7,90 (d, 1H, ArH, $J=$ 1,2), 7,10 (d, 1H, $\mathrm{ArH}, J=1,2$ ), 2,90 (d, $6 \mathrm{H}, \mathrm{CH}_{3}, J=1,6$ ); análise elementar calculada para $\mathrm{C}_{8} \mathrm{H}_{8} \mathrm{Cl}_{2} \mathrm{~N}_{2} \mathrm{O}_{2}: \mathrm{C}, 40,88 ; \mathrm{H}, 3,43 ; \mathrm{N}, 11,92$; encontrada: C, 40,93; H, 3,47; N, 11,90; rendimento: $94 \%$.

1,2-dicloro-4-dietilamino-5-nitrobenzeno, 10: (óleo) RMN de ${ }^{1} \mathrm{H}(200 \mathrm{MHz}) \delta: 7,76$ (s, 1H, ArH), 7,15 (s, 1H, ArH), 3,13 (q, 4H, $\left.\mathrm{CH}_{2}, J=7\right), 1,08\left(\mathrm{t}, 6 \mathrm{H}, \mathrm{CH}_{3}, J=7,1\right)$; rendimento: $93 \%$.

1,2-dicloro-4-di(n-propilamino)-5-nitrobenzeno, 11: (óleo) RMN de ${ }^{1} \mathrm{H}(200 \mathrm{MHz}) \delta: 7,83$ (s, 1H, ArH), 7,18 (s, 1H, ArH), 3,06 (t, 4H, $\left.\mathrm{CH}_{2}, J=7,3\right), 1,54\left(\mathrm{~m}, 4 \mathrm{H}, \mathrm{CH}_{2}\right), 0,86$ (t, 6H, $\mathrm{CH}_{3}, J=$
7,3); rendimento: $91 \%$.

1,2-dicloro-4(n-butilmetilamino)-5-nitrobenzeno, 12: (óleo) RMN de ${ }^{1} \mathrm{H}(200 \mathrm{MHz}) \delta: 7,85$ (s, 1H, Ar-H), 7,12 (s, 1H, Ar-H), 3,15 (t, $\left.2 \mathrm{H}, \mathrm{CH}_{2}, J=7,3\right), 2,81\left(\mathrm{~s}, 3 \mathrm{H}, \mathrm{CH}_{3}\right), 1,59\left(\mathrm{~m}, 2 \mathrm{H}, \mathrm{CH}_{2}\right)$, $1,80\left(\mathrm{~m}, 2 \mathrm{H}, \mathrm{CH}_{2}\right), 0,92\left(\mathrm{t}, 3 \mathrm{H}, \mathrm{CH}_{3}, J=7,2\right)$; rendimento: $87 \%$.

1,2-dicloro-4-pirrolidino-5-nitrobenzeno, 13: p.f. $130{ }^{\circ} \mathrm{C}$; RMN de ${ }^{1} \mathrm{H}(200 \mathrm{MHz}) \delta:$ 7,84 (s, 1H, ArH), 7,01 (s, 1H, ArH), 3,19 (t, $\left.4 \mathrm{H}, \mathrm{CH}_{2}, J=6,4\right), 1,96\left(\mathrm{~m}, 4 \mathrm{H}, \mathrm{CH}_{2}\right)$; análise elementar calculada

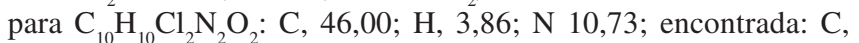
46,09 ; H, 3,96; N, 10,71; rendimento: $93 \%$.

1,2-dicloro-4-piperidino-5-nitrobenzeno, 14: p.f. $62{ }^{\circ} \mathrm{C}$ (Lit. ${ }^{12}$ 104-105 $\left.{ }^{\circ} \mathrm{C}\right)$; RMN de ${ }^{1} \mathrm{H}(200 \mathrm{MHz}) \delta: 7,92$ (s, 1H, ArH), 7,17 (s, $1 \mathrm{H}, \mathrm{ArH}), 3,0\left(\mathrm{~m}, 4 \mathrm{H}, \mathrm{CH}_{2}\right), 1,73\left(\mathrm{~m}, 6 \mathrm{H}, \mathrm{CH}_{2}\right)$; análise elementar calculada para $\mathrm{C}_{11} \mathrm{H}_{12} \mathrm{Cl}_{2} \mathrm{~N}_{2} \mathrm{O}_{2}: \mathrm{C}, 48,02 ; \mathrm{H}, 4,40 ; \mathrm{N}, 10,18$; encontrada: C, 48,12; H, 4,46; N, 10,15; rendimento: $88 \%$.

\section{Procedimento 2: obtenção de produtos cloro-nitro-dissubstituídos}

A um balão de $10 \mathrm{~mL}$ com $0,10 \mathrm{~g}(0,42 \mathrm{mmol})$ de DCDNB, foi adicionada uma solução contendo 4,2 mmol de amina (metilamina, $n$-propilamina, $i$-propilamina, $n$-butilamina, cicloexilamina, dimetilamina, dietilamina, di- $n$-propilamina, piperidina e pirrolidina) em $4 \mathrm{~mL}$ de acetronila. As reações foram realizadas sob refluxo, em atmosfera de nitrogênio, e acompanhadas por cromatografia em camada delgada. Após 24 h de reação, o solvente foi evaporado em rotavapor. Quando um produto predominava, este era recristalizado em etanol e lavado com etanol frio e água. Quando dois produtos principais eram obtidos, a separação era realizada em coluna, utlilizando-se sílica gel 60, empacotada com hexano. Os produtos foram separados por eluição com diclorometano-hexano 1:12 (v/v) e diclorometano-hexano 1:1 (v/v). Após a evaporação do solvente, os produtos foram recristalizados como anteriormente.

1-cloro-2,4-di(metilamino)-5-nitrobenzeno, 15: p.f. $185-186{ }^{\circ} \mathrm{C}$; RMN de ${ }^{1} \mathrm{H}(400 \mathrm{MHz}) \delta: 8,48$ (sl, 1H, NH), 8,15 (d, 1H, ArH, $J=$ 1,2), 5,62 (s, 1H, ArH), 5,16 (sl, 1H, NH), 3,01 (dd, 3H, $\mathrm{CH}_{3}, J=$ 1,2 e 5,2), 2,99 (dd, $3 \mathrm{H}, \mathrm{CH}_{3}, J=1,2$ e 5,6); análise elementar calculada para $\mathrm{C}_{8} \mathrm{H}_{10} \mathrm{ClN}_{3} \mathrm{O}_{2}: \mathrm{C}, 44,56 ; \mathrm{H}, 4,67 ; \mathrm{N}, 19,49$; encontrada: C, 44,63; H, 4,71; N, 19,42; rendimento: $88 \%$.

1-cloro-2,4-di(n-propilamino)-5-nitrobenzeno, 16: p.f. $75-76^{\circ} \mathrm{C}$; RMN de ${ }^{1} \mathrm{H}(200 \mathrm{MHz})$ ä: 8,45 (sl, 1H, NH), 8,16 (s, 1H, ArH), 5,67 (s, 1H, ArH), 5,01 (sl, 1H, NH), 3,21 (m, 4H, $\left.\mathrm{CH}_{2}\right), 1,75(\mathrm{~m}$, $\left.4 \mathrm{H}, \mathrm{CH}_{2}\right), 1,06\left(\mathrm{t}, 6 \mathrm{H}, \mathrm{CH}_{3}, J=7,5\right)$; análise elementar calculada para $\mathrm{C}_{12} \mathrm{H}_{18} \mathrm{ClN}_{3} \mathrm{O}_{2}$ : C, 53, 04; $\mathrm{H} \mathrm{6,68;} \mathrm{N} \mathrm{15,} \mathrm{46;} \mathrm{encontrada:} \mathrm{C}$, 53,$13 ; \mathrm{H}, 6,74 ; \mathrm{N}, 15,44$; rendimento: $26 \%$.

1-cloro-2,4-di(n-butilamino)-5-nitrobenzeno, 17: p.f. $58-59{ }^{\circ} \mathrm{C}$ (Lit. $\left.{ }^{13} 58-58,5{ }^{\circ} \mathrm{C}\right)$; RMN de ${ }^{1} \mathrm{H}(400 \mathrm{MHz}) \delta$ : 8,48 (sl, $\left.1 \mathrm{H}, \mathrm{NH}\right)$, 8,16 (s, 1H, ArH), 5,68 (s, 1H, ArH), 5,01 (sl, 1H, NH), 3,24 (m, $\left.4 \mathrm{H}, \mathrm{CH}_{2}\right), 1,70\left(\mathrm{~m}, 4 \mathrm{H}, \mathrm{CH}_{2}\right), 1,52\left(\mathrm{~m}, 4 \mathrm{H}, \mathrm{CH}_{2}\right), 1,00(\mathrm{~m}, 6 \mathrm{H}$, $\mathrm{CH}_{3}$ ); análise elementar calculada para $\mathrm{C}_{14} \mathrm{H}_{22} \mathrm{ClN}_{3} \mathrm{O}_{2}: \mathrm{C}, 56,09 ; \mathrm{H}$, 7,40 ; N, 14,02; encontrada: C, 56, 12; H, 7,42; N, 14,00; rendimento: $17 \%$.

1-cloro-2,4-bis(dimetilamino)-5-nitrobenzeno, 18: p.f. $89^{\circ} \mathrm{C}$ (Lit. ${ }^{11}$ 90-91 ${ }^{\circ} \mathrm{C}$ ); RMN de ${ }^{1} \mathrm{H}(400 \mathrm{MHz}) \delta: 7,98$ (d, 1H, ArH, $J=2$ ), 6,39 (s, 1H, ArH), 2,94 (d, 3H, $\mathrm{CH}_{3}, J=2$ ), 2,89 (d, 3H, $\mathrm{CH}_{3}, J=2$ ); análise elementar calculada para $\mathrm{C}_{10} \mathrm{H}_{14} \mathrm{ClN}_{3} \mathrm{O}_{2}: \mathrm{C}, 49,29 ; \mathrm{H}, 5,79 ; \mathrm{N}$ 17,24; encontrada: C, 49,36; H, 5,85; N, 17,17; rendimento: $90 \%$. 
1-cloro-2,4-dipirrolidino-5-nitrobenzeno, 19: p.f. $155^{\circ} \mathrm{C}$ (Lit. ${ }^{14}$ $\left.160{ }^{\circ} \mathrm{C}\right)$; RMN de ${ }^{1} \mathrm{H}(200 \mathrm{MHz}) \delta: 7,94$ (s, 1H, ArH), 5,97 (s, 1H, $\mathrm{ArH}), 3,56\left(\mathrm{t}, 4 \mathrm{H}, \mathrm{CH}_{2}, J=6,3\right), 3,21\left(\mathrm{t}, 4 \mathrm{H}, \mathrm{CH}_{2}, J=6,1\right)$ 1,96 (m, $8 \mathrm{H}, \mathrm{CH}_{2}$ ); análise elementar calculada para $\mathrm{C}_{14} \mathrm{H}_{18} \mathrm{ClN}_{3} \mathrm{O}_{2}: \mathrm{C}, 56,85$; $\mathrm{H}, 6,13$; N, 14,21; encontrada: C, 56,89; H, 6,19; N, 14,20; rendimento: $90 \%$.

1-cloro-2,4-dipiperidino-5-nitrobenzeno, 20: p.f. 103-104 ${ }^{\circ} \mathrm{C}$ (Lit. ${ }^{15}$ 103-104 $\left.{ }^{\circ} \mathrm{C}\right)$; RMN de ${ }^{1} \mathrm{H}(200 \mathrm{MHz}) \delta: 8,0$ (s, 1H, ArH), 6,51 (s, 1H, ArH), 3,10 (t, 4H, $\mathrm{CH}_{2}, J=4,4$ e 5,6), 3,99 (t, 4H, $\mathrm{CH}_{2}$, $J=4,5$ e 5,7$), 1,65\left(\mathrm{~m}, 12 \mathrm{H}, \mathrm{CH}_{2}\right)$; análise elementar calculada para $\mathrm{C}_{16} \mathrm{H}_{22} \mathrm{ClN}_{3} \mathrm{O}_{2}: \mathrm{C}, 59,35 ; \mathrm{H}, 6,85 ; \mathrm{N}, 12,98$; encontrada: $\mathrm{C}$, 59,37; H, 6,89; N, 12,94; rendimento: $92 \%$.

\section{Análises cristalográficas}

Os compostos 3, 13 e $\mathbf{2 0}$ tiveram suas estruturas cristalinas/ moleculares determinadas por difração de raios x. A partir de cada amostra cristalina desses compostos foi isolado um monocristal, que foi colocado em difratômetro CAD-4 Enraf-Nonius, usando fonte de Mo e monocromador de grafite, para efetuar a coleta de dados difratométricos em temperatura ambiente. Para as três estruturas, os átomos não-hidrogênio foram refinados anisotropicamente e os átomos de hidrogênio ligados a carbonos foram colocados geometricamente. Na estrutura do composto $\mathbf{3}$, o átomo de hidrogênio da função amina foi localizado a partir do mapa de densidades eletrônicas e tratado como átomo livre. Outras informações sobre as análises cristalográficas são apresentadas na Tabela 1. As tabelas completas dos dados cristalográficos e refinamento foram depositadas no Cambridge Structural Database (números do depósito CCDC 604128, CCDC 604129 e CCDC 604130) e podem ser obtidas, sem qualquer custo, na página da web http://www.ccdc.cam.ac.uk/.

\section{RESULTADOS E DISCUSSÃO}

\section{Substituição do grupo nitro}

O grupo nitro é substituído nas reações envolvendo o DCDNB com aminas primárias e secundárias, produzindo uma série de de-
Tabela 1. Dados cristalográficos e de refinamento selecionados

\begin{tabular}{|c|c|c|c|}
\hline Composto & 3 & 13 & 20 \\
\hline Fórmula & $\mathrm{C}_{9} \mathrm{H}_{10} \mathrm{Cl}_{2} \mathrm{~N}_{2} \mathrm{O}_{2}$ & $\mathrm{C}_{10} \mathrm{H}_{10} \mathrm{Cl}_{2} \mathrm{~N}_{2} \mathrm{O}_{2}$ & $\mathrm{C}_{16} \mathrm{H}_{22} \mathrm{ClN}_{3} \mathrm{O}_{2}$ \\
\hline Peso molecular & 249,09 & $261,10^{2}$ & $323,82^{3}$ \\
\hline$\lambda(\AA)$ & 0,71073 & 0,71073 & 0,71073 \\
\hline Sist. cristalino & Monoclínico & Ortorrômbico & Trigonal \\
\hline Grupo espacial & $\mathrm{P} 2{ }_{1} / \mathrm{c}$ & Pbca & R-3 \\
\hline $\mathrm{a}(\AA)$ & $7,764(2)$ & $7,1634(5)$ & $27,144(2)$ \\
\hline b $(\AA)$ & $17,591(2)$ & $15,358(6)$ & $27,144(2)$ \\
\hline$c(\AA)$ & $7,891(1)$ & $20,2996(14)$ & $12,107(3)$ \\
\hline$\beta\left(^{\circ}\right)$ & $94,48(1)$ & & \\
\hline Volume $\left(\AA^{3}\right)$ & $1074,4(3)$ & $2233,2(8)$ & $7725(2)$ \\
\hline $\mathrm{Z}$ & 4 & 8 & 18 \\
\hline $\begin{array}{l}\text { Densidade calc. } \\
\left(\mathrm{g} / \mathrm{cm}^{3}\right)\end{array}$ & 1,540 & 1,553 & 1,253 \\
\hline $\begin{array}{l}\text { Coef. de absorção } \\
\left(\mathrm{mm}^{-1}\right)\end{array}$ & 0,584 & 0,567 & 0,233 \\
\hline $\mathrm{F}(000)$ & 512 & 1072 & 3096 \\
\hline Médoto de refinament & \multicolumn{3}{|c|}{ Matriz completa com $\mathrm{F}^{2}$} \\
\hline Reflexões coletadas & 2032 & 2081 & 3840 \\
\hline Reflexões únicas & 1887 & 2081 & 3547 \\
\hline Parâmetros refinados & 141 & 146 & 199 \\
\hline $\operatorname{GOOF}\left(\mathrm{F}^{2}\right)$ & 1,036 & 1,035 & 1,069 \\
\hline $\mathrm{R}_{1}[\mathrm{I}>2 \sigma(\mathrm{I})]$ & 0,0421 & 0,0352 & 0,0720 \\
\hline $\mathrm{wR}_{2}$ & 0,1225 & 0,0940 & 0,2300 \\
\hline
\end{tabular}

rivados com rendimentos entre 85 e $95 \%$, exceto o derivado da anilina, cujo rendimento foi de $54 \%$, conforme resultados apresentados na Tabela 2, entradas $\mathbf{1}$ a $\mathbf{1 4}$.

O tempo de reação, medido pelo consumo do DCDNB ou pelo aparecimento da espécie dissubstituída, utilizando cromatografia de camada delgada, variou para cada amina. De modo geral, crescia com o aumento da cadeia alquílica das aminas, o que é devido, principalmente, ao efeito estérico provocado pelas interações das aminas com a cadeia aromática, sem comprometer o efeito ressonante do grupo nitro em orto $^{16}$. As aminas cíclicas, e.g., piperidina e pirrolidina, estão entre as mais nucleofílicas, consumindo o DCDNB, nas condições experimentais estudadas, em no máximo

Tabela 2. Produtos monossubstituídos e dissubstituídos obtidos da reação entre DCDNB e aminas

\begin{tabular}{|c|c|c|c|c|}
\hline Entrada & Amina & Tempo de reação & Condição & rendimento \\
\hline 1 & metilamina & $3 \mathrm{~min}$ & temperatura ambiente & $89 \%$ \\
\hline 2 & $n$-propilamina & $10 \mathrm{~min}$ & temperatura ambiente & $92 \%$ \\
\hline 3 & $i$-propilamina & 1 hora & temperatura ambiente & $95 \%$ \\
\hline 4 & $n$-butilamina & $10 \mathrm{~min}$ & temperatura ambiente & $92 \%$ \\
\hline 5 & $t$-butilamina & $2 \mathrm{~h}$ & refluxo & $94 \%$ \\
\hline 6 & cicloexilamina & $1 \mathrm{~h}$ & temperatura ambiente & $92 \%$ \\
\hline 7 & benzilamina & $8 \mathrm{~h}$ & temperatura ambiente & $85 \%$ \\
\hline 8 & anilina & $4 \mathrm{~h}$ & refluxo & $54 \%$ \\
\hline 9 & dimetilamina & $3 \mathrm{~min}$ & temperatura ambiente & $94 \%$ \\
\hline 10 & detilamina & $16 \mathrm{~h}$ & temperatura ambiente & $93 \%$ \\
\hline 11 & dipropilamina & $2 \mathrm{~h}$ & refluxo & $91 \%$ \\
\hline 12 & $n$-butilmetilamina & $2 \mathrm{~h}$ & refluxo & $87 \%$ \\
\hline 13 & pirrolidina & $3 \mathrm{~min}$ & temperatura ambiente & $93 \%$ \\
\hline 14 & piperidina & $3 \mathrm{~min}$ & temperatura ambiente & $88 \%$ \\
\hline 15 & metilamina & $24 \mathrm{~h}^{\mathrm{a}}$ & refluxo & $88 \%$ \\
\hline 16 & $n$-propilamina & $24 \mathrm{~h}^{\mathrm{a}}$ & refluxo & $26 \%$ \\
\hline 17 & $n$-butilamina & $24 \mathrm{~h}^{\mathrm{a}}$ & refluxo & $17 \%$ \\
\hline 18 & dimetilamina & $24 \mathrm{~h}^{\mathrm{a}}$ & refluxo & $90 \%$ \\
\hline 19 & pirrolidina & $24 \mathrm{~h}^{\mathrm{a}}$ & refluxo & $90 \%$ \\
\hline 20 & piperidina & $24 \mathrm{~h}^{\mathrm{a}}$ & refluxo & $92 \%$ \\
\hline
\end{tabular}

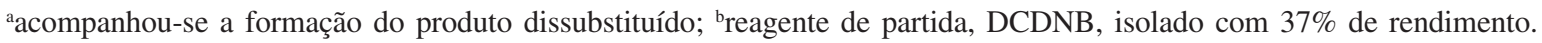


3 min. Amimas mais impedidas, incluindo algumas primárias, todavia, requereram até $24 \mathrm{~h}$, ou mais, de tempo de reação, mesmo sob refluxo (Tabela 2).

As aminas cíclicas são conhecidas por se comportarem de forma diferente das demais aminas secundárias sendo muito mais reativas ${ }^{14}$, como, por ex., nas reações envolvendo a substituição do nitro em 1,2-dinitrobenzeno por aminas ${ }^{17}$.

Diferença em reatividade foi observada até entre aminas cíclicas estruturalmente semelhantes, como no caso da reação do éter etil 2,4dinitronaftila com pirrolidina e piperidina, cuja velocidade de aminólise pela primeira foi 11 mil vezes maior ${ }^{18}$. A anilina foi a menos reativa das aminas, o que é justificado por sua menor força básica.

\section{Substituição dos grupos cloro e nitro}

Após a substituição inicial do grupo nitro, o anel aromático contém ainda três grupos em condições de sofrerem substituição nucleofílica. A segunda substituição, a de um grupo cloro, ocorre muito mais lentamente que a do nitro, conforme esperado para um substrato menos ativado.

Utilizando-se refluxo e 10 vezes excesso do reagente amínico, metilamina, dimetilamina, pirrolidina e piperidina apresentaram reatividade semelhante e converteram o DCDNB nos respectivos produtos de dissubstituição, 15, 19, 20 e 21, em 24 h. As aminas primárias $n$-propilamina e $n$-butilamina, nas mesmas condições, formaram os produtos 16 e 17. As aminas primárias isopropilamina e cicloexilamina, assim como as secundárias dietilamina e dipropilamina, nas mesmas condições, não formaram produtos dissubstituídos, ou o fizeram em quantidade muito pequena.

A acetonitrila foi usada como solvente, devido à sua alta polaridade, o que favorece as substituições nucleofílicas aromáticas ${ }^{19}$, e à facilidade de sua remoção durante o tratamento da reação. Algumas reações foram testadas em clorofórmio, mas mostraram-se muito lentas e em menor rendimento. A variação da velocidade em função da estrutura da amina foi semelhante em todos os solventes.

A substituição de um grupo nitro, em preferência a um cloro, ocorre como esperado de um substrato do tipo do DCDNB, onde o grupo nitro em posição orto ao grupo de saída pode ou deve estabilizar a espécie intermediária por ligação do hidrogênio amínico com os oxigênios ${ }^{5,8,16,20}$. A dissubstituição é, portanto, influenciada pelo grupo nitro que está em posição para ao grupo de saída (cloro).

\section{Caracterização dos produtos}

Os produtos dessas reações são, quase todos, sólidos, de coloração laranja-intensa. As análises de $\mathrm{RMN}$ de ${ }^{1} \mathrm{H}, \mathrm{CHN}$ e os valores de ponto de fusão dos compostos conhecidos permitiram que as estruturas dos produtos fossem caracterizadas. Todavia, a posição exata da substituição, especialmente, nos produtos dissubstituídos, foi reconhecida por cristalografia de raios $\mathrm{X}$, conforme mostrado nas estruturas referentes aos compostos $\mathbf{3}, \mathbf{1 3}$ e 20, obtidos como monocristais próprios para análise cristalográfica após sucessivas recristalizações, e apresentados na Figura 1.

\section{CONCLUSÕES}

O DCDNB reage em condições suaves com aminas primárias e secundárias produzindo produtos de substituição do grupo nitro com rendimentos entre 85 e 95\%. A segunda substituição, do cloro, ocorre mais lentamente e com menores rendimentos. Os reagentes

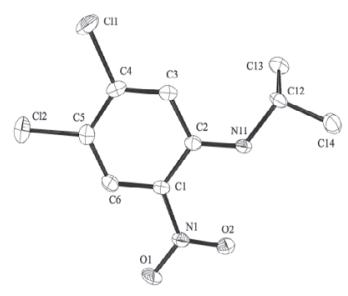

3

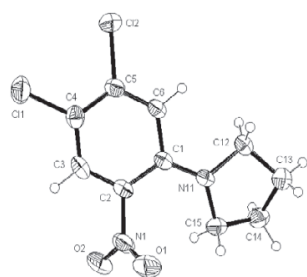

13

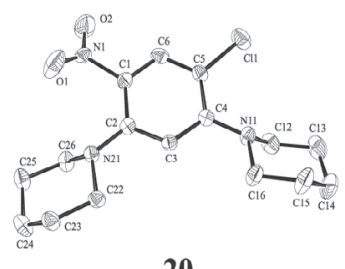

20

Figura 1. Representação espacial das estruturas moleculares dos compostos 3, 13 e 20. Os elipsóides estão representados com probabilidade de $40 \%$

amínicos piperidina, pirrolidina, dimetilamina e metilamina foram os mais reativos tanto na mono quanto na dissubstituição.

\section{AGRADECIMENTOS}

Ao apoio financeiro da UFSC, da FINEP, da CAPES e do CNPq às pesquisas e às bolsas de estudo e pesquisa fornecidas.

\section{REFERÊNCIAS}

1. March, J.; Advanced Organic Chemistry, $3^{\text {rd }}$ ed., Wiley: New York, 1985.

2. Lowry, T. H.; Richardson, K. S.; Mechanism and Theory in Organic Chemistry, $3^{\text {rd }}$ ed., Harper \& Row Publishers: New York, 1987.

3. Zucco, C.; trabalho não publicado.

4. Bacaloglu, R.; Blasko, A.; Bunton, C.; Dorvin, E.; Ortega, F.; Zucco, C.; J. Am. Chem. Soc. 1991, 113, 238; Bacaloglu, R.; Blasko, A.; Bunton, C.; Ortega, F.; Zucco, C.; J. Am. Chem. Soc. 1992, 114, 7708.

5. Bradshaw, J. D.; Tessier, C. A.; Youngs, W. J.; Tetrahedron Lett. 1995, 36, 6217.

6. Zucco, C.; Neves, A.; Vencato, I.; Szpoganicz, B.; Bertoldi, F.C.; Acta Crystallogr., Sect. C: Cryst. Struct. Commun. 1999, 55, 654.

7. Beck, J. R. Tetrahedron 1978, 34, 2057.

8. Pietra, F.; Vitali, D.; J. Chem. Soc., Perkin Trans. 2 1972, 2, 385.

9. Feitelson, B. N.; Mamalis, P.; Moualim, R. J.; Petrow, V.; Stephenson, O.; Sturgeon, B.; J. Chem. Soc. 1952, 2389. (CA 47:1132f).

10. Oezden, S.; Karatas, H.; Yildiz, S.; Goeker, H.; Arch. Pharmacal. Res. 2004, 337, 556.

11. Woolley, D. W.; Pringle, A.; J. Biol. Chem. 1952, 194, 729.

12. Lefevre, R. J. W.; Turner, E. E.; J. Chem. Soc. 1927, 1113. (CA 21:2681).

13. Fevre, C. W. L.; Hunter, D. L.; Woods, W. G.; DE2058225, 1972. (CA 77:P126204c)

14. Ibata, T.; Isogami, Y.; Toyoda, J.; Bull. Chem. Soc. Jpn. 1991, 64, 42.

15. Seikel, M. K.; J. Am. Chem. Soc. 1940, 62, 750.

16. Pietra, F.; Cima, F. D.; J. Org. Chem. 1968, 33, 1411; Pietra, F.; Vitalli, D.; Cima, F. D.; Cardinalli, G.; J. Chem. Soc., B 1970, 9, 1659.

17. Catttana, R.I.; Singh, J. O.; Anunziata, J. D.; Silber, J. J.; J. Chem. Soc., Perkin Trans. 2 1987, 1, 79.

18. Bunnett, J. F.; Sekigushi, S.;Smith, A. L.; J. Am Chem Soc. 1981, 103, 4865.

19. Reichardt, C.; Solvents and solvents effects in organic chemistry 2; rev. And enl. Ed, VCH: New York, 1988.

20. Ayediran, D.;Bamkole, T. O.; Hirst, J.; Onyido, I.; J. Chem. Soc., Perkin Trans 2. 1977, 5, 597; Bernasconi, C.F.; Rossi, R. H. D.; J. Org. Chem. 1976, 41, 44. 